Open J. Math. Anal., Vol. 2(2018), No. 2, pp. 41 - 50

Website: https://pisrt.org/psr-press/journals/oma/

ISSN: 2616-8111 (Online) 2616-8103 (Print)

http://dx.doi.org/10.30538/psrp-oma2018.0016

\title{
TOPOLOGICAL DEGREES ON UNBOUNDED DOMAINS
}

\author{
DHRUBA R. ADHIKARI ${ }^{1}$, ISHWARI J. KUNWAR
}

\begin{abstract}
Let $D$ be an open subset of $\mathbf{R}^{N}$ and $f: \bar{D} \rightarrow \mathbf{R}^{N}$ a continuous function. The classical topological degree for $f$ demands that $D$ be bounded. The boundedness of domains is also assumed for the topological degrees for compact displacements of the identity and for operators of monotone type in Banach spaces. In this work, we follow the methodology introduced by Nagumo for constructing topological degrees for functions on unbounded domains in finite dimensions and define the degrees for LeraySchauder operators and $\left(S_{+}\right)$-operators on unbounded domains in infinite dimensions.

Mathematics Subject Classification: Primary 47H14; Secondary 47H05, $47 \mathrm{H} 11$.

Key words and phrases: Brouwer degree; Leray-Schauder degree; Browder and Skrypnik degrees; Unbounded domains; Bounded demicontinuous operators of type $\left(S_{+}\right)$.
\end{abstract}

\section{Introduction}

The theory of topological degrees has progressed significantly in recent years because of its applicability to the analysis of ordinary and partial differential equations and continuation methods in nonlinear analysis in general (e.g. see [1, 2, 3, 4, 5, 6, 7, 8, 9]). The classical topological degree theory developed by Brouwer [10] in 1912 for continuous functions on finite-dimensional spaces and the Leray-Schauder degree [1] in 1934 for compact displacements of the identity in Banach spaces both assume the boundedness of the domains over which the degrees are defined. There are numerous generalizations and extensions of

Received 21-09-218. Revised 07-10-2018. Accepted 09-10-2018.

1 Corresponding Author

(c) 2018 Dhruba R. Adhikari and Ishwari J. Kunwar. This is an open access article distributed under the Creative Commons Attribution License, which permits unrestricted use, distribution, and reproduction in any medium, provided the original work is properly cited. 
these degree theories all of which are expressed, one way or the other, in terms of the Brouwer degree or the Leray-Schauder degree, and therefore the boundedness of domains becomes an essential consideration. Nagumo [12] defined the Brouwer degree based on infinitesimal analysis and indicated the possibility for developing degree theories over unbounded domains. In this paper, we construct topological degrees on unbounded domains both in finite-dimensional and infinite dimensional spaces and discuss their properties. For the development of degree theories for operators of monotone type that involve $\left(S_{+}\right)$-operators and their generalized and/or multivalued versions, the reader is referred to Kartsatos and Skrypnik 1, 2, Berkovits [13, Berkovits and Mustonen [14, Kartsatos and the first author [5, 7, Kartsatos and Kerr [8, Hu and Papageorgiou [15, Kittilä [16] and the references therein. For the coincidence degree developed by Mawhin for nonlinear perturbations of certain Fredholm operators in normed spaces, the reader is referred to [17.

In Section 2 we elaborate on the Nagumo's definition of the Brouwer degree on unbounded domains by verifying the assertions made in [12. We then prove a version of the Leray-Schauder lemma for this new degree. In Section 3 , we construct the Leray-Schauder degree on unbounded domains and discuss its desirable properties. Section 4 deals with the degree theory on unbounded domains for operators of type $\alpha_{0}\left(S_{+}\right)$introduced by Skrypnik [18].

\section{Brouwer Degree on Unbounded Domains}

Let $D \subset \mathbf{R}^{N}$ be open and $f: \bar{D} \rightarrow \mathbf{R}^{N}$ continuous. Let $\mathscr{D}$ be the set of all sequences in $D$ that have no limit points in $D$. Define the set $f\{D\}$ to be the set of all limit points of the sequence $\left\{f\left(x_{n}\right)\right\}$ for $\left\{x_{n}\right\} \in \mathscr{D}$. It follows that $f(\partial D) \subset f\{D\}$. In fact, let $p \in f(\partial D)$. Then there exist $x_{0} \in \partial D$ and $\left\{x_{n}\right\}$ in $D$ such that $f\left(x_{0}\right)=p$ and $x_{n} \rightarrow x_{0}$. The continuity of $f$ implies $f\left(x_{n}\right) \rightarrow f\left(x_{0}\right)=p$. Since $x_{0} \notin D$, it follows that $\left\{x_{n}\right\} \in \mathscr{D}$, and therefore $p \in f\{D\}$. The inclusion $f(\partial D) \subset f\{D\}$ may be strict in general (e.g. for $N=1$, let $D=(1, \infty), f(x)=1 / x$. Then $f(\partial D)=\{1\}$ and $f\{D\}=\{0,1\})$; however, if $D$ is bounded, then $f\{D\}=f(\partial D)$. To prove this, let $q \in f\{D\}$. Then there exists $\left\{x_{n}\right\} \in \mathscr{D}$ such that $\left\{f\left(x_{n}\right)\right\}$ has a subsequence $\left\{f\left(x_{n_{k}}\right)\right\}$ such that $f\left(x_{n_{k}}\right) \rightarrow q$ as $k \rightarrow \infty$. Since $D$ is bounded, we may assume that $\left\{x_{n_{k}}\right\}$ converges to $x_{0} \in \bar{D}$. Since $\left\{x_{n}\right\} \in \mathscr{D}$, we have $x_{0} \in \partial D$, and therefore $f\left(x_{0}\right)=q$, i.e. $q \in f(\partial D)$.

Suppose now that $D$ is unbounded, and assume that $p \notin f\{D\}$ for the same $f$ as above. Denote

$$
R=\{x \in D: f(x)=p\} .
$$

We can show that $R$ is closed and bounded in $\mathbf{R}^{N}$. In fact, let $\left\{x_{n}\right\}$ be a sequence in $R$ such that $x_{n} \rightarrow x_{0}$ for some $x_{0} \in \mathbf{R}^{N}$. Then $f\left(x_{n}\right)=p$ for all $n$, and $x_{0} \in \bar{D}$. If $x_{0} \in \partial D$, then $\left\{x_{n}\right\} \in \mathscr{D}$ so that $p \in f\{D\}$, a contradiction. Thus, $x_{0} \in D$. Since $f\left(x_{0}\right)=p$, we have $x_{0} \in R$, and therefore $R$ is closed. In order to show that $R$ is bounded, suppose the contrary and let $\left\{x_{n}\right\}$ be a sequence in $R$ such 
that $\left\|x_{n}\right\| \rightarrow \infty$. This implies that $\left\{x_{n}\right\} \in \mathscr{D}$. However, since $x_{n} \in D$ and $f\left(x_{n}\right)=p$ for all $n$, we have $p \in f\{D\}$, which is a contradiction. Now, let $D_{1}$ and $D_{2}$ be any two bounded open sets such that $R \subset D_{i}$ and $\bar{D}_{i} \subset D$ for $i=1,2$. Then $f$ is continuous on $\bar{D}_{i}, p \notin f\left(\partial D_{i}\right)$ and the Brouwer's degree, $\mathrm{d}_{\mathrm{B}}\left(f, D_{i}, p\right)$, is well-defined for $i=1,2$. Also, since there is no solution of $f(x)=p$ in $\left(\bar{D}_{1} \backslash R\right) \cup\left(\bar{D}_{2} \backslash R\right)$, we have

$$
\mathrm{d}_{\mathrm{B}}\left(f, D_{1}, p\right)=\mathrm{d}_{\mathrm{B}}\left(f, D_{2}, p\right) .
$$

We define the degree of $f$ at $p$ over $D$ as

$$
\mathrm{d}(f, D, p)=\mathrm{d}_{\mathrm{B}}\left(f, D_{0}, p\right),
$$

where $D_{0}$ is any bounded open set containing $R$ and $\bar{D}_{0} \subset D$.

The degree mapping defined in (1) has the following properties, usually called the desirable properties of any degree:

(i) Let $I$ be the identity function. Then $\mathrm{d}(I, D, p)=1$ if $p \in D$ and $p \notin$ $I\{D\}$, and $\mathrm{d}(I, D, p)=0$ if $p \notin \bar{D}$. Note that $I\{D\}=\partial D$, and therefore it may be empty.

(ii) If $\mathrm{d}(f, D, p) \neq 0$, then there exists $x \in D$ such that $f(x)=p$.

(iii) If $D \supset \bigcup_{i=1}^{n} D_{i}$, where $D_{i}$ 's are mutually disjoint open sets such that $\bar{D}=\bigcup_{i=1}^{n} \bar{D}_{i}$ and $p \notin f\{D\}$, then

$$
p \notin \bigcup_{i=1}^{n} f\left\{D_{i}\right\} \text { and } \mathrm{d}(f, D, p)=\sum_{i=1}^{n} \mathrm{~d}\left(f, D_{i}, p\right) .
$$

(iv) (Homotopy Invariance) If $f(t, x)$ is a continuous on $[0,1] \times \bar{D}$ and $p$ : $[0,1] \rightarrow \mathbf{R}^{N}$ is continuous such that $p(t) \notin f\left(t ; t_{0}\right)\{D\}$ for all $t \in[0,1]$ and $t_{0} \in[0,1]$ (see the paragraph below for notations used here), then $\mathrm{d}(f(t, \cdot), D, p(t))$ is constant for all $t \in[0,1]$.

We will only prove the homotopy invariance property. Let $f:[0,1] \times \bar{D} \rightarrow \mathbf{R}^{N}$ be a continuous mapping, and let $f\left(t ; t_{0}\right)\{D\}$ denote the set of all limit points of $\left\{f\left(t_{n}, x_{n}\right)\right\}$, where $\left\{t_{n}\right\} \subset[0,1], t_{n} \rightarrow t_{0}$, and $\left\{x_{n}\right\} \in \mathscr{D}$. As before, if $D$ is bounded, then $f\left(t ; t_{0}\right)\{D\}=f\left(\left\{t_{0}\right\} \times \partial D\right)$.

Lemma 2.1. Let $f:[0,1] \times \bar{D} \rightarrow \mathbf{R}^{N}$ be continuous, $t_{0} \in[0,1]$ be fixed, and $p \notin f\left(t ; t_{0}\right)\{D\}$. Then there exist a neighborhood $U$ of $p$, a number $\delta>0$ and a bounded open set $D_{0}$ such that $\bar{D}_{0} \subset D$ and

$$
U \cap f\left(t, D \backslash D_{0}\right)=\emptyset
$$

for all $t \in\left(t_{0}-\delta, t_{0}+\delta\right)$.

Proof. Assume that the conclusion does not hold. For each $n \in \mathbb{N}$, define

$$
D_{n}=\{x \in D:\|x\|<n \text { and } \operatorname{dist}(x, \partial D)>1 / n\} .
$$

Then each $D_{n}$ is open and bounded. It is also clear that $\bar{D}_{n} \subset D$ for all $n$. By the assumption, for each $n \in \mathbb{N}$, there exist $y_{n} \in \mathbf{R}^{N}$ and $t_{n} \in[0,1]$ such that 
$t_{n} \rightarrow t_{0}$ and

$$
y_{n} \in B(p ; 1 / n) \cap f\left(t_{n}, D \backslash D_{n}\right) .
$$

Then there exists $x_{n} \in D \backslash D_{n}$ such that $f\left(t_{n}, x_{n}\right)=y_{n} \rightarrow p$. Since $x_{n} \notin D_{n}$, we have either $\left\|x_{n}\right\| \geq n$ or $\operatorname{dist}\left(x_{n}, \partial D\right) \leq 1 / n$. If $\left\|x_{n}\right\| \geq n$ for infinitely many values of $n$, then we may assume that $\left\{x_{n}\right\} \in \mathscr{D}$, and so $p \notin f\left(t ; t_{0}\right)\{D\}$, which is a contradiction. Therefore $\operatorname{dist}\left(x_{n}, \partial D\right) \leq 1 / n$ for infinitely many values of $n$. If $\left\{x_{n}\right\}$ has a subsequence $\left\{x_{n_{k}}\right\}$ such that $x_{n_{k}} \rightarrow x_{0}$ for some $x_{0} \in \mathbf{R}^{N}$, then $\operatorname{dist}\left(x_{n_{k}}, \partial D\right) \rightarrow \operatorname{dist}\left(x_{0}, \partial D\right)=0$, which implies $x_{0} \in \partial D$. Since $f$ is continuous, we have $f\left(t_{0}, x_{0}\right)=p$ so that $p \in f\left(t_{0}, \partial D\right)$ which contradicts $p \notin f\left(t ; t_{0}\right)\{D\}$ because $f\left(t_{0}, \partial D\right) \subset f\left(t ; t_{0}\right)\{D\}$. This means that $\left\{x_{n}\right\}$ has no convergent subsequence, and therefore $\left\{x_{n}\right\} \in \mathscr{D}$. However, this implies $p \notin f\left(t ; t_{0}\right)\{D\}$, which is again a contradiction. This completes the proof.

Proposition 2.2. Let $f:[0,1] \times \bar{D} \rightarrow \mathbf{R}^{N}$ be continuous, and assume that, for a fixed $t_{0} \in[0,1], p(t) \notin f\left(t ; t_{0}\right)\{D\}$ for all $t \in[0,1]$. Then there exist number $\delta>0$, an open set $U$ and a bounded open set $D_{0}$ with $\bar{D}_{0} \subset D$ such that

$$
p(t) \in U \quad \text { and } \quad U \cap f\left(t, D \backslash D_{0}\right)=\emptyset
$$

for all $t \in\left(t_{0}-\delta, t_{0}+\delta\right)$.

Proof. Since $p\left(t_{0}\right) \notin f\left(t ; t_{0}\right)\{D\}$, by Lemma 2.1 there exist a neighborhood $U$ of $p\left(t_{0}\right)$, a number $\delta>0$ and a bounded open set $D_{0}$ such that

$$
\bar{D}_{0} \subset D \text { and } U \cap f\left(t, D \backslash D_{0}\right)=\emptyset
$$

for all $t \in\left(t_{0}-\delta_{1}, t_{0}+\delta\right)$. The continuity of $p$ ensures that we can shrink $\delta$, if necessary, so that $p(t) \in U$ for all $t \in\left(t_{0}-\delta, t_{0}+\delta\right)$.

Theorem 2.3 (Homotopy Invariance). Let $f:[0,1] \times \bar{D} \rightarrow \mathbf{R}^{N}$ be continuous, and let $p:[0,1] \rightarrow \mathbf{R}^{N}$ be continuous such that, for every $t_{0} \in[0,1], p(t) \notin$ $f\left(t ; t_{0}\right)\{D\}$ for all $t \in[0,1]$. Then the degree $\mathrm{d}(f(t, \cdot), D, p(t))$ is a constant for all $t \in[0,1]$.

Proof. In the settings of Proposition 2.2, we have $\partial D_{0}=\bar{D}_{0} \backslash D_{0} \subset D \backslash D_{0}$ and $U \cap f\left(t, D \backslash D_{0}\right)=\emptyset$ for all $t \in\left(t_{0}-\delta, t_{0}+\delta\right)$, and therefore $p(t) \notin f\left(t, \partial D_{0}\right)$. This implies

$$
\mathrm{d}(f(t, \cdot), D, p(t))=\mathrm{d}_{\mathrm{B}}\left(f(t, \cdot), D_{0}, p(t)\right)
$$

for all $t \in\left(t_{0}-\delta, t_{0}+\delta\right)$. Since $[0,1]$ is compact, by applying a standard covering argument we find that the degree $\mathrm{d}(f(t, \cdot), D, p(t))$ is constant for all $t \in[0,1]$.

We next give a version of the Leray-Schauder lemma [18, Lemma 1.1] for the Brouwer degree on unbounded domains. If $x=\left(x_{1}, \ldots, x_{N-1}, x_{N}\right) \in \mathbf{R}^{N}$, we write $x=\left(x^{\prime}, x_{N}\right)$, where $x^{\prime}=\left(x_{1}, \ldots, x_{N-1}\right) \in \mathbf{R}^{N-1}$. 
Theorem 2.4. Let $D \subset \mathbf{R}^{N}$ be an unbounded open set and $0 \in D$. Let $f: \bar{D} \rightarrow$ $\mathbf{R}^{N}$ be continuous with $f=\left(f_{1}, \ldots, f_{N}\right)$ and satisfy

$$
f_{N}(x) \equiv x_{N} \text { for } x=\left(x^{\prime}, x_{N}\right) \in D .
$$

Suppose that $0 \notin f\{D\}$ and $D^{\prime}=\left\{x^{\prime}:\left(x^{\prime}, 0\right) \in D\right\}$ is nonempty. Then

$$
\mathrm{d}(f, D, 0)=\mathrm{d}\left(f^{\prime}, D^{\prime}, 0\right),
$$

where $f^{\prime}: \overline{D^{\prime}} \rightarrow \mathbb{R}^{N-1}$ defined by

$$
f^{\prime}\left(x^{\prime}\right)=\left(f_{1}\left(x^{\prime}, 0\right), \ldots, f_{N-1}\left(x^{\prime}, 0\right)\right) .
$$

Proof. We assert that $0 \notin f^{\prime}\left\{D^{\prime}\right\}$. Otherwise, there would exist a sequence $\left\{u_{n}^{\prime}\right\} \in \mathscr{D}^{\prime}$ such that $f^{\prime}\left(u_{n}^{\prime}\right) \rightarrow 0$. This implies that $f_{i}\left(u_{n}^{\prime}, 0\right) \rightarrow 0$ for each $i=1, \ldots, N-1$. Let $v_{n}=\left(u_{n}^{\prime}, 0\right)$. Then $f_{N}\left(v_{n}\right)=0$ and $\left\{v_{n}\right\} \in \mathscr{D}$ and $f\left(v_{n}\right) \rightarrow 0$. This implies $0 \in f\{D\}$, a contradiction.

Next, let $D_{0} \subset \mathbf{R}^{N}$ be a bounded open set containing $R=\{x \in D: f(x)=0\}$ such that $\bar{D}_{0} \subset D$. Since $x \in R$ implies $x_{N}=0$, we define $R^{\prime}=\left\{x^{\prime} \in \mathbf{R}^{N-1}\right.$ : $x \in R\}$. Then $R^{\prime}=\left\{x^{\prime} \in D^{\prime}: f^{\prime}\left(x^{\prime}\right)=0\right\}$, and therefore $D_{0}^{\prime}:=\left\{x^{\prime}: x \in D_{0}\right\}$ is a bounded open set in $\mathbf{R}^{N-1}$ satisfying $R^{\prime} \subset D_{0}^{\prime}$ and $\overline{D^{\prime}}{ }_{0} \subset D^{\prime}$. Applying the Leray-Schauder lemma [18, Lemma 1.1], we see that

$$
\mathrm{d}_{B}\left(f, D_{0}, 0\right)=\mathrm{d}_{B}\left(f^{\prime}, D_{0}^{\prime}, 0\right) .
$$

Since these degrees are independent of the choice of $D_{0}$, by the definition of the degree in (1), we have

$$
\mathrm{d}(f, D, 0)=\mathrm{d}\left(f^{\prime}, D^{\prime}, 0\right) .
$$

\section{Leray-Schauder Degree on Unbounded Domains}

Let $X$ be a Banach space, $D$ an open subset of $X$ and $T: \bar{D} \rightarrow X$ a compact operator ( $T$ is continuous and maps bounded sets to relatively compact sets). When $D$ is bounded, the construction of the Leray-Schauder degree for the compact displacement of the identity over $D$ uses the fact due to Schauder that each compact operator on a bounded domain is the uniform limit of a sequence of compact operators of finite-dimensional range. This consideration relies on the boundedness of $D$ so that $\overline{T(D)}$ is compact. However, if $D$ is unbounded, the approximation scheme is not applicable. In this section, we give a definition of the degree of $I-T$ when $D$ is unbounded.

As in Section 1, we define the set $(I-T)\{D\}$ as the set of limit points of the sequence $\left\{x_{n}-T x_{n}\right\}$ for $\left\{x_{n}\right\} \in \mathscr{D}$, where $\mathscr{D}$ has the same meaning as in Section 1. One can easily verify that $(I-T)(\partial D) \subset(I-T)\{D\}$. However, if $D$ is bounded, then it follows that $(I-T)(\partial D)=(I-T)\{D\}$. In fact, suppose $D$ is bounded, and let $p \in(I-T)\{D\}$. Then there exists $\left\{x_{n}\right\} \in \mathscr{D}$ such that $x_{n}-T x_{n} \rightarrow p$. Since $D$ is bounded and $T$ is compact, we may assume (up to a subsequence) that $T x_{n} \rightarrow y_{0}$ for some $y_{0} \in X$. This implies that 
$x_{n} \rightarrow y_{0}+p=: x_{0}$, say. By the definition of $\mathscr{D}$, we must have $x_{0} \in \partial D$. The continuity of $T$ yields $T x_{n} \rightarrow T x_{0}$, and therefore $p=x_{0}-T x_{0} \in(I-T)(\partial D)$. For a given point $p \in X$ such that $p \notin(I-T)\{D\}$, we now proceed to give a meaning to the degree mapping $\mathrm{d}(I-T, D, p)$. Suppose $p \notin(I-T)\{D\}$, and define $R=\{x \in D: x-T x=p\}$. We can easily show that $R$ is bounded and closed in $X$. Let $D_{i}, i=1,2$, be bounded open sets such that $R \subset D_{i} \subset D$.

Since $x-T x=p$ has no solutions in $\left(\bar{D}_{1} \backslash R\right) \cup\left(\bar{D}_{2} \backslash R\right)$, the Leray-Schuader degree, $\mathrm{d}_{\mathrm{LS}}$, satisfies

$$
\mathrm{d}_{\mathrm{LS}}\left(I-T, D_{1}, p\right)=\mathrm{d}_{\mathrm{LS}}\left(I-T, D_{2}, p\right) .
$$

We define

$$
\mathrm{d}(I-T, D, p)=\mathrm{d}_{\mathrm{LS}}\left(I-T, D_{0}, p\right),
$$

where $D_{0}$ is any bounded open set such that $R \subset D_{0} \subset D$.

We now proceed to consider suitable homotopies under which this new degree is invariant as in Proposition 3.

Lemma 3.1. Let $p \notin(I-T)\{D\}$. Then there exist a neighborhood $U$ of $p$ and a bounded open set $D_{0}$ such that $\bar{D}_{0} \subset D$ and

$$
U \cap(I-T)\left(D \backslash D_{0}\right)=\emptyset .
$$

Proof. Assume that the conclusion does not hold. For each $n \in \mathbb{N}$, define $D_{n}$ as in Lemma 2.1. Then $D_{n}$ is open and bounded. It is also clear that $\bar{D}_{n} \subset D$ for all $n$. By the assumption, for each $n \in \mathbb{N}$, there exists

$$
y_{n} \in B(p ; 1 / n) \cap(I-T)\left(D \backslash D_{n}\right) .
$$

Then, for each $n \in \mathbb{N}$, there exists $x_{n} \in D \backslash D_{n}$ such that $x_{n}-T x_{n}=y_{n} \rightarrow p$. Since $x_{n} \notin D_{n}$, either $\left\|x_{n}\right\| \geq n$ or $\operatorname{dist}\left(x_{n}, \partial D\right) \leq 1 / n$. If $\left\|x_{n}\right\| \geq n$ for infinitely many values of $n$, then we may assume that $\left\{x_{n}\right\} \in \mathscr{D}$, and so $p \in(I-T)\{D\}$, which is a contradiction. Therefore $\operatorname{dist}\left(x_{n}, \partial D\right) \leq 1 / n$ for infinitely many values of $n$. If $\left\{x_{n}\right\}$ has a subsequence $\left\{x_{n_{k}}\right\}$ such that $x_{n_{k}} \rightarrow x_{0}$ for some $x_{0} \in X$, then $\operatorname{dist}\left(x_{n_{k}}, \partial D\right) \rightarrow \operatorname{dist}\left(x_{0}, \partial D\right)=0$, which implies $x_{0} \in \partial D$. Since $T$ is compact, we have $x_{0}-T x_{0}=p$ so that $p \in(I-T)(\partial D)$, a contradiction. This means that $\left\{x_{n}\right\}$ has no convergent subsequence, and therefore $\left\{x_{n}\right\} \in \mathscr{D}$. However, this also implies $p \in(I-T)\{D\}$, a contradiction.

Let $T:[0,1] \times \bar{D} \rightarrow X$ be a compact mapping, and let $(I-T)\left(t ; t_{0}\right)\{D\}$ denote the set of all limit points of $\left\{x_{n}-T\left(t_{n}, x_{n}\right)\right\}$, where $\left\{t_{n}\right\} \subset[0,1], t_{n} \rightarrow t_{0}$, and $\left\{x_{n}\right\} \in$ $\mathscr{D}$. As before, if $D$ is bounded, then $(I-T)\left(t ; t_{0}\right)\{D\}=(I-T)\left(\left\{t_{0}\right\} \times \partial D\right)$.

In view of Lemma 3.1, we can prove an analog of Lemma 2.1 which we only state as follows.

Lemma 3.2. Let $T:[0,1] \times \bar{D} \rightarrow X$ be compact and $t_{0} \in[0,1]$ be fixed. Let $p \notin(I-T)\left(t ; t_{0}\right)\{D\}$. Then there exist a neighborhood $U$ of $p$, a number $\delta>0$ and $a$ bounded open set $D_{0}$ such that $\bar{D}_{0} \subset D$ and

$$
U \cap(I-T)\left(t, D \backslash D_{0}\right)=\emptyset
$$


for all $t \in\left(t_{0}-\delta, t_{0}+\delta\right)$.

Theorem 3.3 (Homotopy Invariance). Let $T:[0,1] \times \bar{D} \rightarrow X$ be compact, and let $p:[0,1] \rightarrow \mathbf{R}^{N}$ be continuous such that, for all $t_{0} \in[0,1], p(t) \notin$ $(I-T)\left(t ; t_{0}\right)\{D\}$ for all $t \in[0,1]$. Then the degree $\mathrm{d}(f(t, \cdot), D, p(t))$ is constant for all $t \in[0,1]$.

Proof. For each fixed $t_{0} \in[0,1]$ as in Lemma 3.2 , we have $\partial D_{0}=\bar{D}_{0} \backslash D_{0} \subset$ $D \backslash D_{0}$ and $U \cap(I-T)\left(t, D \backslash D_{0}\right)=\emptyset$ for all $t \in\left(t_{0}-\delta, t_{0}+\delta\right)$, and therefore $p(t) \notin(I-T)\left(t, \partial D_{0}\right)$. This implies

$$
\mathrm{d}(I-T(t, \cdot), D, p(t))=\mathrm{d}_{\mathrm{LS}}\left(I-T(t, \cdot), D_{0}, p(t)\right)
$$

for all $t \in\left(t_{0}-\delta, t_{0}+\delta\right)$. Since $[0,1]$ is compact, by applying the Borel's covering argument we find that the degree $\mathrm{d}(I-T(t, \cdot), D, p(t))$ is constant for all $t \in[0,1]$.

\section{Degree for $\left(S_{+}\right)$-Operators on Unbounded Domains}

In what follows, $X$ denotes a real separable reflexive Banach space and $X^{*}$ its dual. Without loss of generality, we assume that $X$ is locally uniformly convex. In this setting, the normalized duality mapping $J$ from $X$ to $X^{*}$ is single-valued, bicontinuous and strongly maximal monotone operator given by

$$
J x=\left\{x^{*} \in X^{*}:\left\langle x^{*}, x\right\rangle=\|x\|^{2},\left\|x^{*}\right\|=\|x\|\right\} .
$$

The mapping $J$ plays crucial roles in establishing existence results for operator equations that involve operators of monotone type in Banach spaces (cf. [5, 13, 19, 15, 1, 18]). A more general class of operators is given in the definition below.

Definition 4.1. Let $D \subset X$ be open. An operator $T: \bar{D} \rightarrow X^{*}$ is said to be of class $\alpha_{0}\left(S_{+}\right)$if each sequence $\left\{u_{n}\right\}$ in $\bar{D}$ with $u_{n} \rightarrow u_{0}$ in $X, T u_{n} \rightarrow 0$ in $X^{*}$ and

$$
\limsup _{n \rightarrow \infty}\left\langle T u_{n}, u_{n}-u_{0}\right\rangle \leq 0
$$

is in fact strongly convergent to $u_{0}$.

The operators of class $\alpha_{0}\left(S_{+}\right)$were first studied by Skrypnik [18] and are more general than $\left(S_{+}\right)$-operators considered by Browder [19, Berkovits [13, and several other authors.

Let $T: \bar{D} \rightarrow X^{*}$ be a bounded demicontinuous of type $\alpha_{0}\left(S_{+}\right)$, and let $T\{D\}$ be the set of all weak limits of $\left\{T u_{n}\right\}$ where $\left\{u_{n}\right\} \in \mathscr{D}$, where $\mathscr{D}$, as in previous sections, denotes the set of all sequences in $D$ that have no limit points in $D$. We want to make sure that the set $T\{D\}$ contains $T(\partial D)$. In fact, let $p \in T(\partial D)$. Then $p=T u_{0}$ for some $u_{0} \in \partial D$, and therefore there exists a sequence $\left\{u_{n}\right\} \in \mathscr{D}$ such that $u_{n} \rightarrow u_{0}$. By the demicontinuity of $T$, we get $T u_{n} \rightarrow T u_{0}=p$, which implies $p \in T\{D\}$. Thus, $T(\partial D) \subset T\{D\}$. One can verify that when $D$ is bounded and $T$ maps bounded sets to relatively compact sets, then $T(\partial D)=T\{D\}$. 
We proceed to define the degree, $\mathrm{d}(T, D, 0)$, of a bounded demicontinuous mapping $T$ of type $\alpha_{0}\left(S_{+}\right)$under the condition $0 \notin T\{D\}$ when $D$ is unbounded. In this setting, let $R=\{x \in D: T x=0\}$. As in the previous sections, $R$ is bounded and closed in $X$ here as well. In fact, let $x_{0} \in \bar{R}$. Then there exists a sequence $\left\{x_{n}\right\} \subset D$ with $T x_{n}=0$ such that $x_{n} \rightarrow x_{0}$. Since $T$ is demicontinuous on $\bar{D}, T x_{n} \rightarrow T x_{0}=0$. If $x_{0} \in D$, we are done. Otherwise, $x_{0} \in \partial D$. Then $x_{0} \in T(\partial D) \subset T\{D\}$, a contradiction. Thus, $R$ is closed in $X$. To show the boundedness of $R$, suppose that there exists a sequence $\left\{x_{n}\right\}$ in $R$ such that $\left\|x_{n}\right\| \rightarrow \infty$. Clearly, $\left\{x_{n}\right\} \in \mathscr{D}$ and $T x_{n}=0$ for all $n$. This contradicts $0 \notin T\{D\}$, and therefore $R$ must be bounded. Choose a bounded open set $U$ such that $R \subset U \subset D$.

Theorem 4.2. Let $D \subset X$ be an unbounded open set and $T: \bar{D} \rightarrow X^{*}$ a bounded demicontinuous mapping of type $\alpha_{0}\left(S_{+}\right)$. Assume $0 \notin T\{D\}$. Then there exists $n_{0} \in \mathbb{N}$ such that $0 \notin T_{n}\left(\partial U_{n}\right)$ for all $n \geq n_{0}$ and the degree $\mathrm{d}_{\mathrm{B}}\left(T_{n}, U_{n}, 0\right)$ is defined. Moreover, the degree $\mathrm{d}_{\mathrm{B}}\left(T_{n}, U_{n}, 0\right)$ is independent of $n$.

Proof. Since $0 \notin T\{D\}$, there exists a bounded open subset $U$ of $X$ such that $R \subset U \subset D$, where $R=\{x \in D: T x=0\}$. It now follows that $0 \notin T(\partial U)$.

Let $\left\{v_{i}\right\}, i=1,2, \ldots$, be a complete system of $X$. Suppose that $\left\{v_{1}, \ldots, v_{n}\right\}$ is linearly independent for every $n$, and let $F_{n}=\operatorname{span}\left\{v_{1}, \ldots, v_{n}\right\}$. We now define the finite-dimensional approximation $T_{n}$ of $T$ as

$$
T_{n} u=\sum_{i=1}^{n}\left\langle T u, v_{i}\right\rangle v_{i} \quad \text { for } u \in \bar{U}_{n}, \quad U_{n}=U \cap F_{n} .
$$

For each $n, U_{n}$ is a bounded open subset of $F_{n}$, and $T_{n}: \bar{U}_{n} \rightarrow F_{n}$ is a continuous operator. We now apply Theorem 2.1 in [18, p.35] to obtain the rest of the conclusion.

Remark 4.3. It would be interesting to determine whether a version of Theorem 4.2 may be given by directly using the approximations $T_{n}$ defined on $\bar{D}_{n}$, where $D_{n}=D \cap F_{n}$. The set $D_{n}$ is open in $F_{n}$, but it may be unbounded. In this case, the degree theory introduced in Section 2 may be used to compute $\mathrm{d}\left(T_{n}, D_{n}, 0\right)$. A suitable condition replacing $0 \notin T\{D\}$ in Theorem 4.2 is needed. Such a condition must be contradicted by the condition that $0 \in T_{n_{k}}\left\{\mathscr{D}_{n_{k}}\right\}$ for a sequence $\left\{n_{k}\right\}$ of positive integers with $n_{k} \rightarrow \infty$ as $k \rightarrow \infty$. Here, $\mathscr{D}_{n_{k}}$ has the same meaning as that of $\mathscr{D}$ in Section 2, A version of the Leray-Schuader lemma given in Theorem 2.4 may be useful to establish the independence of $\mathrm{d}\left(T_{n}, D_{n}, 0\right)$ for sufficiently large $n$.

\section{Acknowledgement}

The first author sincerely acknowledges the support from the College of Science and Mathematics at Kennesaw State University through the 2018 Research Stimulus Program during the summer of 2018 for carrying out this research work. The collaborative work began effectively during the authors' participation in 
the Workshop on Collaborative Research in Mathematical Sciences organized by Association of Nepalese Mathematicians in America (ANMA) during May 25 27, 2018, in the Department of Mathematics at Mercer University, Georgia. The authors are grateful to anonymous reviewer(s) for providing invaluable feedback for the improvement of the paper.

\section{Competing Interests}

The authors declare that they have no competing interests.

\section{REFERENCES}

1. Kartsatos, A. G., \& Skrypnik, I. V. (1999). Topological degree theories for densely defined mappings involving Operators of Type $\left(\mathrm{S}_{+}\right)^{*}$. Advances in Differential Equations, 4(3), 413-456.

2. Kartsatos, A. G., \& Skrypnik, I. V. (2005). A new topological degree theory for densely defined quasibounded $\left(\tilde{S}_{+}\right)$-perturbations of multivalued maximal monotone operators in reflexive Banach spaces. Abstr. Appl. Anal., Volume 2005(2), 121-158.

3. Adhikari, D. R., \& Kartsatos, A. G. (2008). Topological degree theories and nonlinear operator equations in Banach spaces. Nonlinear Analysis: Theory, Methods 83 Applications, 69(4), 1235-1255.

4. Kartsatos, A. G., \& Quarcoo, J. (2008). A new topological degree theory for densely defined $\left(S_{+}\right)_{L^{-}}$perturbations of multivalued maximal monotone operators in reflexive separable Banach spaces. Nonlinear Analysis: Theory, Methods $\& 3$ Applications, 69(8), 2339-2354.

5. Adhikari, D. R., \& Kartsatos, A. G. (2008). Strongly quasibounded maximal monotone perturbations for the BerkovitsMustonen topological degree theory. Journal of Mathematical Analysis and Applications, 348(1), 122-136.

6. Ibrahimou, B., \& Kartsatos, A. G. (2009). The LeraySchauder approach to the degree theory for $\left(S_{+}\right)$-perturbations of maximal monotone operators in separable reflexive Banach spaces. Nonlinear Analysis: Theory, Methods 86 Applications, 70(12), 4350-4368.

7. Adhikari, D. R., \& Kartsatos, A. G. (2011). A new topological degree theory for perturbations of the sum of two maximal monotone operators. Nonlinear Analysis: Theory, Methods ES Applications, 74(14), 4622-4641.

8. Kartsatos, A. G., \& Kerr, D. (2011). A Browder degree theory from the Nagumo degree on the Hilbert space of elliptic super-regularization. Nonlinear Analysis: Theory, Methods ES Applications, 74(2), 501-515.

9. Asfaw, T. M., \& Kartsatos, A. G. (2012). A Browder topological degree theory for multivalued pseudomonotone perturbations of maximal monotone operators. Advances in Mathematical Sciences and Applications, 22(1), 91-148.

10. Brouwer, L. E. J. (1911). Über abbildung von mannigfaltigkeiten. Mathematische Annalen, $71(1), 97-115$

11. Leray, J., \& Schauder, J. (1934). Topologie et équations fonctionnelles. Ann. Sci. Ecolé Norm. Sup., 51(3), 45-78.

12. Nagumo, M. (1951). A theory of degree of mapping based on infinitesimal analysis. American Journal of Mathematics, 73(3), 485-496.

13. Berkovits, J. (1986). On the degree theory for nonlinear mappings of monotone type(Doctoral dissertation). Ann. Acad. Sci. Fenn. Ser. A I Math., 58.

14. Berkovits, J., \& Mustonen, V. (1990). On the topological degree for perturbations of linear maximal monotone mappings and applications to a class of parabolic problems. Rend. Mat. Appl., Serie VII 12, 597-621.

15. Hu, S. C., \& Papageorgiou, N. S. (1995). Generalizations of Browders degree theory. Trans. Amer. Math. Soc., 347(1), 233-259. 
16. Kittilä, A. (1994). On the topological degree for a class of mappings of monotone type and applications to strongly nonlinear elliptic problems(Doctoral dissertation). Ann. Acad. Sci. Fenn. Ser. A I Math., 91.

17. Gaines, R. E., \& Mawhin, J. L. (2006). Coincidence degree and nonlinear differential equations. Springer-Verlag, New York.

18. Skrypnik, I. V. (1994). Methods for analysis of nonlinear elliptic boundary value problems. American Mathematical Society.

19. Browder, F. E. (1983). Fixed point theory and nonlinear problems. Bull. Amer. Math. Soc., 9(1), 1-39.

\section{Dhruba R. Adhikari}

Department of Mathematics, Kennesaw State University, Georgia 30060, USA.

e-mail: dadhikar@kennesaw.edu

Ishwari J. Kunwar

Department of Mathematics and Computer Science, Fort Valley State University, Georgia 31030, USA.

e-mail: kunwari@fvsu.edu 